\title{
Der Neuromarkt
}

\author{
E. Taverna
}

Im Lichthof der Universität nehmen Frauen und Männer ihre Plätze ein. Sie legen neue Broschüren auf die Tische, stapeln Flyers und Magazine und locken die Besucher mit Süssigkeiten und Kunstblumen an ihre Stände. Die Trennwände informieren über die Ziele der Betroffenenorganisationen und Gesellschaften. «Fragile» schildert die Welt der Hirnverletzten, die Liga gegen Epilepsie präsentiert einen Comic zum Gewitter im Hirn, auf dem Platzspitz werden Jogger für MS-Betroffene ihre Runden drehen, Elternvereine erklären was POS-Kinder sind und der Schleudertraumaverband ist mit Ratschlägen zur Hand. Vereine wie Equilibrium zur Bewältigung von Depressionen stellen sich vor, ebenso fundiert wie die Arbeitsgemeinschaft für Aphasie, der Gehörlosenbund, die Vereinigungen für Parkinson oder Alzheimer und viele andere mehr. Das angebotene Informationsmaterial ist vielseitig, gut verständlich und meist auch graphisch ansprechend. Im Rahmen der «Internationalen Woche des Gehirns» hat das Zentrum für Neurowissenschaften Zürich mit den erwähnten Organisationen die «Brain Fair 2002» gestaltet.

Podiumsdiskussionen, Vorträge, Filme, aber auch Tanz und Theater suchen den Gedankenaustausch mit der eingeladenen Bevölkerung. Mediengespräche, Tage der offenen Türen, Programme für die Schulen und Demonstrationen sorgen für eine gute Präsenz in allen Tageszeitungen. Der Markt wird auch nächstes Jahr wieder abgehalten. Lohnt es sich für Mediziner hinzugehen?

\section{Die Krankheiten ...}

Die Schau möchte Brücken schlagen zwischen Laien und Fachpersonen, zwischen Steuerzahlern und Forschung. Was die Institute leisten, verblüfft und fasziniert jeden, der nicht selber forscht. Da ist der Wissensabstand zwischen Journalisten, Ärzten und Patienten nicht mehr gross. Die Mediziner haben den Vorteil, in Krankheitskategorien zu denken und können dabei auf pathophysiologische Grundkenntnisse aufbauen. Sie kommen auf ihre Rechnung, wenn im Keller der Neurologischen Klinik Freiwillige in einem 3-D-Drehstuhl auf das Labyrinth des Innenohrs untersucht werden. Ob High-Tech im Schwindellabor, Stammzellforschung oder Expe- rimente zu geschlechtsabhängigen Unterschieden des Gehirns, die Neuigkeiten aus der Hirnforschung sind beste Fortbildung und Horizonterweiterung, ganz unabhängig vom eigenen FMH-Titel. Auch für diese Aspekte ist jeweils reichlich Lektüre vorhanden: die Übersichtsartikel und Magazine der «European Dana Alliance for the Brain» oder die zahlreichen Unterlagen der beteiligten neurologischen Institute und klinischen Abteilungen, wie das Paraplegikerzentrum vom Balgrist, die Psychiatrieforschung oder das Epilepsiezentrum. Die Wachstumsförderung von Nervenfasern der Ratte und die Computeranalyse des Sehsystems der Wüstenameise werden eines Tages unseren Praxisalltag beeinflussen, genauso wie die künstliche Netzhaut auf dem Siliziumchip oder der mobile Samurairoboter die Klinik bereits verändern.

\section{... und Kranke}

Es gibt für Mediziner noch einen Grund, hinzugehen. Man könnte diesen, etwas romantisch, mit Herzensbildung umschreiben. Der Kontakt mit Patienten aus der Sicht der Betroffenen und Pflegenden entwickelt das Gemüt. Die Diskussionen und die dazugehörigen Filme ergänzen das notwendige Kopfwissen. In diesem Jahr sind es einfühlsame Berichte über die Betreuung und Lebensgestaltung von an Alzheimer erkrankten Menschen. Reportagen vom schleichenden Verfall mit seinen täglichen Niederlagen und Misserfolgen, von der inneren Verlorenheit in einer Welt der «kognitiven Schutzlosigkeit». Porträts von Hirnverletzten, die mühsam ihren Bewegungsraum neu definieren müssen. Tapfer ertragene Schicksale, aus denen sogar Humor aufblitzt, wenn der Alltag mit listigen Tricks und Tips bewältigt wird. Einsichten, die unter die Haut gehen: «Wir helfen uns im Anderssein» oder «Der Kopf hilft sich mit Bildern, bei denen das Wesentliche im Dunkeln bleibt.» Wie bewegt sich ein Mensch in einer Bahnhofshalle, wenn er nur noch die eine Köperhälfte wahrnimmt, wie schlage ich einen Nagel in die Wand, wenn die eine Hand gelähmt ist, wo liegt das Problem, wenn meine Behinderung äusserlich gar nicht sichtbar ist? Die Kamera bringt uns die Einzelschicksale ausserhalb der Sprechstunde näher und gibt Perspektiven frei, wie sie nur durch 
intensive Nähe entstehen können. Das Konzept der Ausstellungen vereinigt mit seinem breit gefächerten Angebot alle Ebenen des Helfens. Das ist wohl ihr grösstes Verdienst.
Wenn in der gleichen Woche Inserate behaupten: «Tierversuche garantieren, dass MS unheilbar bleibt» ortet auch der Sympathisant gravierende Defizite. Auch das ist ein Hinweis, warum es diese Veranstaltungen braucht.

\title{
Ein Plädoyer für Medizinische Labortechnik in Entwicklungsländern
}

\author{
C. Eberle*
}

\footnotetext{
* Die Autorin ist Cheflaborantin/ CLS im Ruhestand und ist zurzeit in folgenden Bereichen tätig: ETH/NADEL Weiterbildungskurse Entwicklungszusammenarbeit; UNIZ: Islamwissenschaften und Konfliktforschung
}

\section{Einführung}

Angesichts der modernen hochtechnisierten Medizin in den Wohlstandsländern ist es den armen Ländern nicht zu verübeln, dass sie auch davon profitieren möchten. Manche Entwicklungshelfer, Ökonomen und Beamte haben Probleme mit sogenannter «Spitzenmedizin» in Drittweltländern. Viel lieber wäre ihnen übers Land verteilte Dispensarien mit präventivmedizinisch ausgebildeten Gesundheitshelfern, welche Menschen aufklären über Hygiene, Infektionskrankheiten, Ernährung, Familienplanung und Malaria. Im besten Fall ein Buschspitälchen mit einem «allround doctor around the clock», der das Allernotwendigste auf einfache und billige Weise erledigt, einen Krankenpfleger, der Medikamente und Spritzen verabreicht und auch einmal eine Wunde versorgen könnte. Manche Verantwortliche meinen, nur Präventivmassnahmen sind sinnvoll.

Nein, weit gefehlt! Nicht nur Vorbeugung, auch die Behandlung der anstehenden Probleme sind wichtiger Beitrag zur Verbesserung der Gesundheit, damit verbunden eine Armutsreduktion. Es versteht sich von selbst, dass dies nur zusammen mit einer Verbesserung der wirtschaftlichen Lage zum Erfolg führen kann. Eine erfolgreiche Behandlung ist möglich, wenn die Diagnose (Fragestellung) einer Krankheit sicher erhoben werden kann. Unter anderem ist dies erreichbar mit richtiger, rascher labormedizinischer Diagnosetechnik. Eine rasche, genaue klinische und labortechnische Diagnose hilft nicht nur der Entscheidungsfindung des Arztes, sie hilft auch die Kosten zu senken, da gezielt das richtige Medikament eingesetzt werden kann, ohne eine Vielzahl teurer Arzneimittel auszuprobieren. Dies ist in der Dritten Welt häufig nur schwer umzusetzen, da entweder keine Therapierichtlinien vorhanden sind oder, falls «Guidelines» vorhanden sind, diese nur beschränkt befolgt werden (klassisches Beispiel: TBC-Therapie). Gleichzeitig können Folgeschäden durch falsch verabreichte Medikamente vermieden werden. Bei der $\mathrm{Zu}$ nahme von komplizierten, kombinierten Krankheitsbildern ist es unabdingbar für den Arzt, diese frühzeitig einzuschätzen, bevor Schaden entstanden ist. Auch dies ist nur mit Hilfe medizinischer Labortechniken möglich.

Eine neue sanfte, technisierte Labordiagnostik einzuführen, die vor allem der Landbevölkerung sowie den Menschen in städtisch-wirtschaftlichen Agglomerationen zugute kommt, macht durchaus Sinn, umso mehr, als sie mit wenig Aufwand, unter Einbezug eigener Kräfte bewerkstelligt werden kann, nicht zu vergessen die damit verbundenen Ausbildungsmöglichkeiten.

Dies ist ein sehr hohes Ziel, eine stetige Gratwanderung zwischen Basismedizin und einer westlich orientierten «High-Tech»-Medizin.

Es gilt klar zu unterscheiden zwischen zwei verschiedenen Arten von Gesundheitszentren (Spitälern) mit verschiedenen Bevölkerungsstrukturen und verschiedenen Bedürfnissen. Dies sind zum einen die Landspitäler (rural hospital), meist mausarm. Sollte trotzdem einmal ein Hilfspaket auf den Weg geschickt werden, dann versinkt die Gabe schnell im Dschungel der Bürokratie und Korruption. 
Zum anderen die Spitäler in Agglomerationen, die finanzielle bessere Möglichkeiten aufweisen. Dort ist es durchaus möglich, die Bevölkerung an einem ökonomischen Konzept zu beteiligen, z.B. an einem partnerschaftlichen «Umlageverfahren», indem Zahlungsfähige die Behandlung der Armen mitfinanzieren, die Armen bezahlen wenig, Halbprivate und Private bezahlen mehr. Unternehmen und Gewerbetreibende bezahlen Krankenkosten für ihre Angestellten, dann gäbe es noch die Möglichkeit für interessierte Private, jährliche Anteilscheine zu zeichnen. Last but not least kann mit einem Labor Geld erwirtschaftet werden, z.B. Ärzte aus der Agglomeration können ihre Bestimmungen im Spitallabor durchführen lassen.

\section{Poverty Reduction Strategy Paper}

Es lohnt sich, einen Blick in den Leitfaden des PRSP-Papier zu werfen. PRSP ist ein Instrument, entwickelt von EZA (Entwicklungszusammenarbeit) und Weltbank, gerichtet an die Regierungen der betreffenden Empfängerländer zur Reduktion der Armut sowie zur Förderung des Wirtschaftswachstums:

- nur wachstumsfokussierte Politik kann Armut reduzieren;

- nur armutsfokussierte Politik kann allgemeines Wachstum produzieren.

Die Federführung für die Strategieentwicklung liegt nach wie vor bei der Regierung des betreffenden Empfängerlandes. Bilaterale Donatoren sind eingeladen, ihre Beiträge in die Umsetzung dieser Strategie einzuspeisen. In 60 Ländern ist der Prozess angelaufen oder in Vorbereitung.

Schaut man sich den mehrseitigen Aktionsplan, der das Gesundheitswesen betrifft, an, wird ersichtlich, wie wichtig der Kampf für die Gesundheit zur Reduktion der Armut eingestuft wird. Klar ist auch, welche Anstrengungen seitens der Regierung verlangt werden, nämlich den vorgegebenen Zeitplan einzuhalten. Es ist auch klar, dass ohne erhebliche Donatorenhilfe aus dem Norden das vorgeschriebene Ziel nie erreicht werden kann, was zur Folge hat, dass die Weltbankgelder versiegen. In welchem Zeitraum und in welchem Umfang werden die bilateralen Donatoren bereit sein, ihre Unterstützung auch tatsächlich in den PRSP-Prozess einzubringen?

\section{Hauptziel}

In dem mir zur Verfügung stehenden Strategiepapier (PRSP) an die anvisierte Gesundheitsbehörde der Republik Mosambik lautet der Auftrag in Kurzform wie folgt:

- Vermeiden von Epidemien (Durchfall);

- Reduktion der Kindersterblichkeit;

- Früherkennung und Behandlung (Lepra);

- Vorsorge und Vorbeugung (Malaria),

- Aufklärung und freiwilliger Test (HIV/AIDS);

- Kampf der TBC und deren Übertragung ...;

- Verbesserung der Geburtshilfe und Nachsorge der Mütter (von mir hinzugefügt);

- Verbesserung der Gesundheitszentren und Spitäler;

- besserer Zugang zu den Zentren, bessere geographische Verteilung;

- Ausbildung von Personal.

Labordiagnostische Aspekte dazu

Labordiagnostik ist sinnvoll und möglich bei folgenden Krankheitsbildern:

\begin{tabular}{|c|c|c|}
\hline & $\begin{array}{l}\text { einfache } \\
\text { Techniken } \\
\text { (rural) }\end{array}$ & $\begin{array}{l}\text { erweiterte } \\
\text { Techniken } \\
\text { (Agglomeration) }\end{array}$ \\
\hline Durchfallerkrankungen & keine / (+) & ++ \\
\hline Malaria & ++ & ++ \\
\hline Tuberkulose* & keine / (+) & ++ \\
\hline HIV/Aids & $(+)$ & ++ \\
\hline Lepra & + & + \\
\hline$($ Hepatitis)** & keine / (+) & ++ \\
\hline
\end{tabular}

(+) Schnelltest möglich, + gute einfache Techniken, ++ sehr gute exakte Methoden (von mir angefügt).

* Keine Diagnostik, wenn nicht eine umfassende Therapie entsprechend den Landesrichtlinien gewährleistet ist. Ansonsten nur Prävention der Übertragung.

** Enzymdiagnostik.

So weit so gut, besser als gar nichts, aber trotzdem nur einen Tropfen auf einen heissen Stein. Sicher, die Grundbedürfnisse der Bevölkerung sind damit abgedeckt, nur das Spektrum zur Verbesserung der Lebensqualität, somit auch zur Armutsreduktion ist viel grösser.

Zunehmende zivilisatorische Krankheitsbilder, sei es als primäres Geschehen oder als Folgeerscheinung, sind leider im Vormarsch. Diesen Krankheitsbildern lässt sich nicht mehr mit einfacher «Buschlabordiagnostik» (Mikroskop) auf den Leib rücken, dazu sind weiterentwickelte labortechnische Methoden (z.B. Nass- oder Trockenchemie) erforderlich, jedoch keine komplizierten softwaregesteuerten Apparate. 
Von der Chirurgie wird schon gar nicht gesprochen. In Afrika und den meisten Entwicklungsländern sind die meisten Patienten viel jünger als bei uns. Angeborene Fehlbildungen (Klumpfüsse, krumme Beine, andere Deformationen) und Infektionskrankheiten, auch am Knochen (Tuberkulose), sind sehr häufig. Oft werden die Kinder und Jugendlichen nicht oder zu spät behandelt. Beängstigend ist die steigende Zahl von Verkehrsunfällen. Die Patienten mit diesen Verletzungen kommen oft zu spät ins Spital; Knocheninfektionen, daraus resultierende Amputationen und Invalidität sind die Folgen. Unfallverletzungen, wenn nicht professionell behandelt, führen zu Verkrüppelung, Arbeitsunfähigkeit ist vorprogrammiert, für die meisten bedeutet dies Armut. In den Industrieländern hat sich als Therapie der Wahl von schweren Knochenbrüchen ein chirurgischer Eingriff (Osteosynthese) etabliert, um die Verletzten baldmöglichst wieder an den Arbeitsplatz zurückzubringen. Nicht so in den armen Ländern des Südens, ihnen fehlen die Geldmittel für sterile Operationssäle, geschultes Personal, Anästhesie und Labor (z. B. Blutgasanalysen).

Die Kritiker steigen sicherlich schon wieder auf die Barrikaden, aber ich erinnere noch einmal an den eingangs geschilderten Wunsch der Menschen aus den armen Ländern: «Es ist den armen Ländern nicht zu verübeln, dass sie auch von unserer technisierten Medizin profitieren möchten. Barfuss- oder Luxusmedizin, weder noch, sondern ein Mittelmass von beidem.»

Mit Humor, von Herzen geschrieben, wohlwissend, dass es wie bei «Don Quijote» ein stetiger Kampf gegen Windmühlen sein wird. 